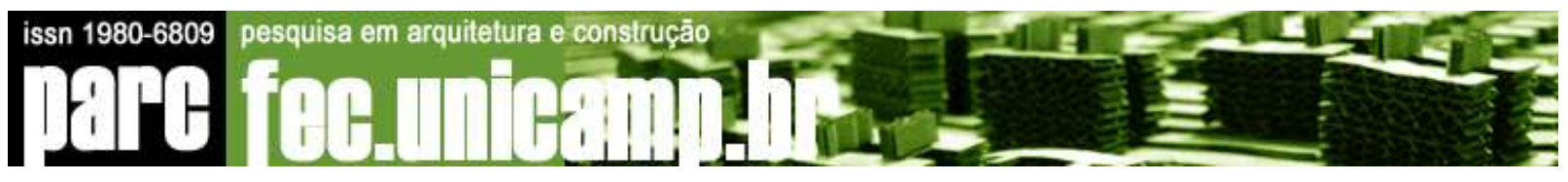

\title{
Computational design contributions of integrative architectural and urban digital design methodology based on satellite images
}

\author{
STELIOS TH. KOUZELEAS
}

Architect, bachelor in Architecture school of Aristotle University of Thessaloniki in Greece, Master of architectural acoustic digital design and simulation in Architecture school of Bordeaux in France, Ph.D. in architectural digital design and acoustic simulation in University of Bordeaux 1 in France, Researcher - Collaborator of GRECO research center of architecture school of Bordeaux in France, Professor Lecturer of digital design and spatial simulation in the Department of urban planning and development engineering of the Aristotle University of Thessaloniki in Greece.

stelios_kouzeleas@yahoo.fr

\begin{abstract}
This study is based on innovative researches and combines technology, methodologies and means which are used in different sciences and processes such as informatics and computational processes, telegeoprocessing-telegeomonitoring technologies, survey science methodologies, urban and regional planning techniques in order to describe computational processes of innovative digital integrative design. This procedure refers to aspects of digital integrative design - modeling and simulation of a built-up architectural or urban area. These aspects concern a modeling process based on satellite images and specifically developed computational interfaces adapted to a CAD system, such as DTM's mesh control points, conversion from geodetic to cartesian coordinates, bitmap adjustment to the buildings facades and surfaces normals handling, taking into account techniques that refer to other sciences such as survey, maths, astronomy and computer science. This modeling process is supported by an innovated proposed procedure that transfers remotely spatial data collected from the field (geographical coordinates and relative measurements taken in place) directly into a modeling system in order to model architectural entities and simulate simultaneously qualitative characteristics of an urban space (sound, temperature, humidity, etc) in real-time.
\end{abstract}

Keywords: Urban digital design technology, CAD, Architectural digital design, Digital spatial simulation, Computational design process. 


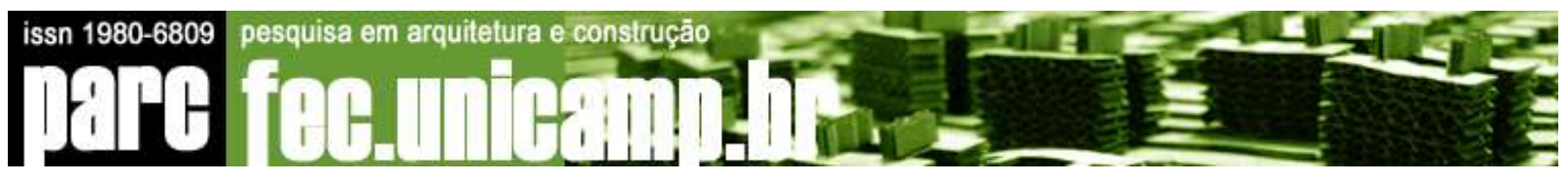

\section{Computational design contributions of integrative architectural digital design methodology based on satellite images}

\section{Introduction}

Over the past years, a collaborative approach to design is more and more emergent and developed. This is due to the implication of multidisciplinary fields and particularly the increased participation of new technologies to design. The design in architecture is influenced progressively from computer science and it is adapted to new processes and technologies concerning similar or other domains such as urban regional planning science, survey science, engineering, physics, etc. The introduction of new widespread technologies changes progressively the process of design and influences, as one goes along, even the design conception of the architectural and urban area. Thus, the architectural and urban design must appropriate, discover and adapt to innovative processes, techniques and technologies in different disciplines; this "opportunistic" approach of design creates collaboration, "fusion" of knowledge and it can be referred to as "integrative design".

The present study takes advantage of innovative techniques and data (such as satellite images, coordinates conversion methods and GPS data), and supplementary wide spread technologies (such as GPS, VHF telecommunications and field sensors) and methodologies that are commonly used in telegeoprocessing - telegeomonitoring in order to establish processes of dynamic digital design with interoperability between them.

The procedure of integrative architectural digital design is based on satellite imagery as geographical background in order to vectorize the architectural entities using adequately converted coordinates taken from satellite images by using a specially developed conversion interface adapted to a CAD system. Based on these geographical coordinates, qualitative characteristics of the space (sound, temperature, humidity, etc) can be modeled and simulated simultaneously as transparent parallel layers. The procedure in question can be

supported voluntarily by a system based on contemporary techniques of geosciences (Laurini, 2000, 1-12) involving telecommunication means (such as GPS, VHF, telecommunications, digital sensors, etc) particularly useful for remote monitoring and real-time applications (Tanzi, 2000, 13-24) in order to facilitate spatial data transfer, processing and representation directly from the field (real space) to the office (in virtual modeling environments such as CAD and GIS) in real-time. 


\section{Methodology}

The methodology concerns a process of different steps involving, in each one, applications, specific interfaces, techniques and technological means (FIG.1). In the first step, the topographic map of the study area must be extracted in satellite image format (e.g. from the Google Earth environment) and inserted in CAD environment without any scale changes.

In the second step, the study area must be digitized and vectoring in CAD environment (AutoCAD) in order to model the parcels and the properties of the building blocks in 2D vectors.

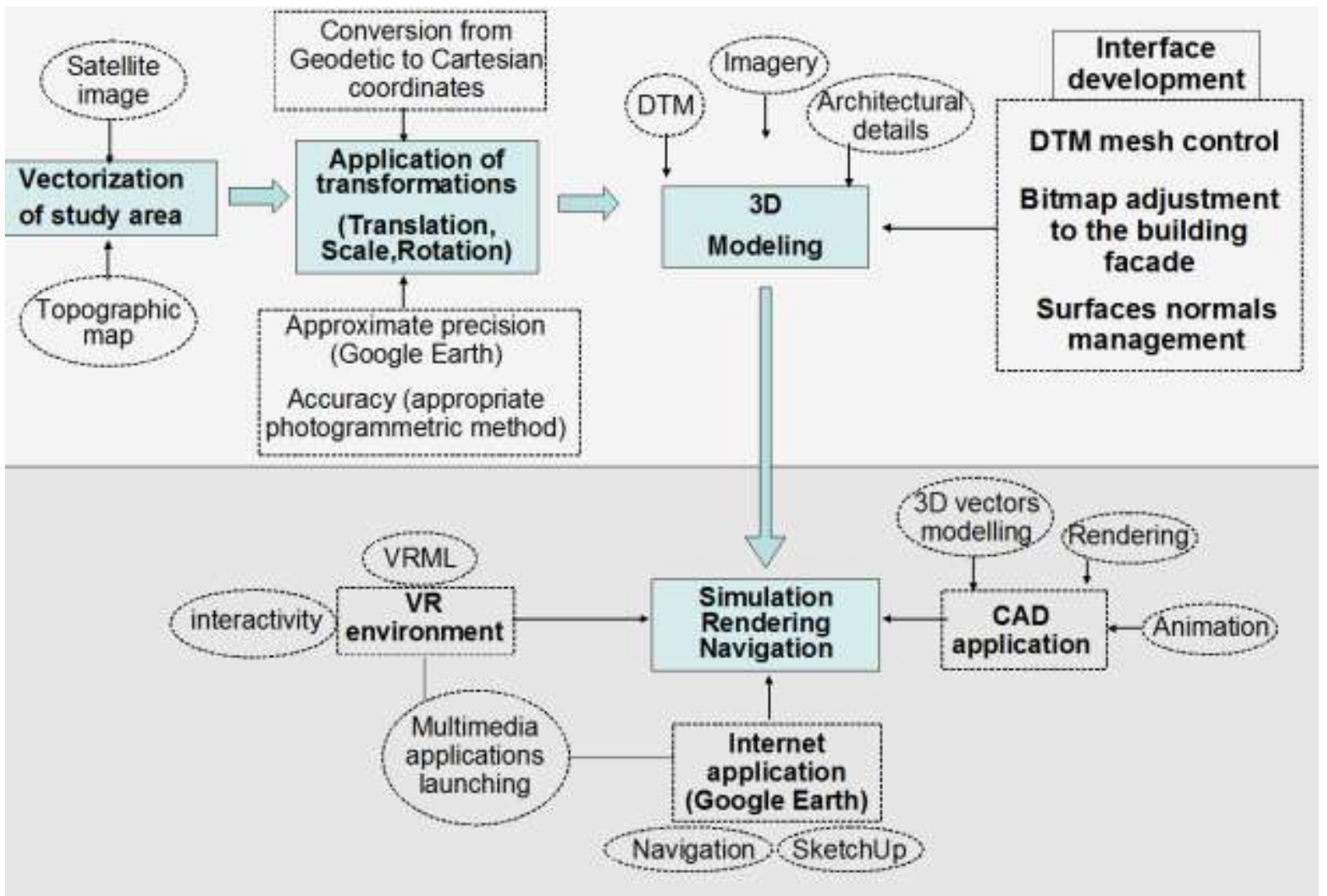

FIGURE 1: Schema of the procedure phases of integrative architectural digital design.

In the third step, in the vectoring environment (2D vectors), the transformations of translation, scale and rotation must be applied in order to adjust the virtual vectoring environment and the satellite imagery to the dimensions and the geographical position of reality (FIG.2). 


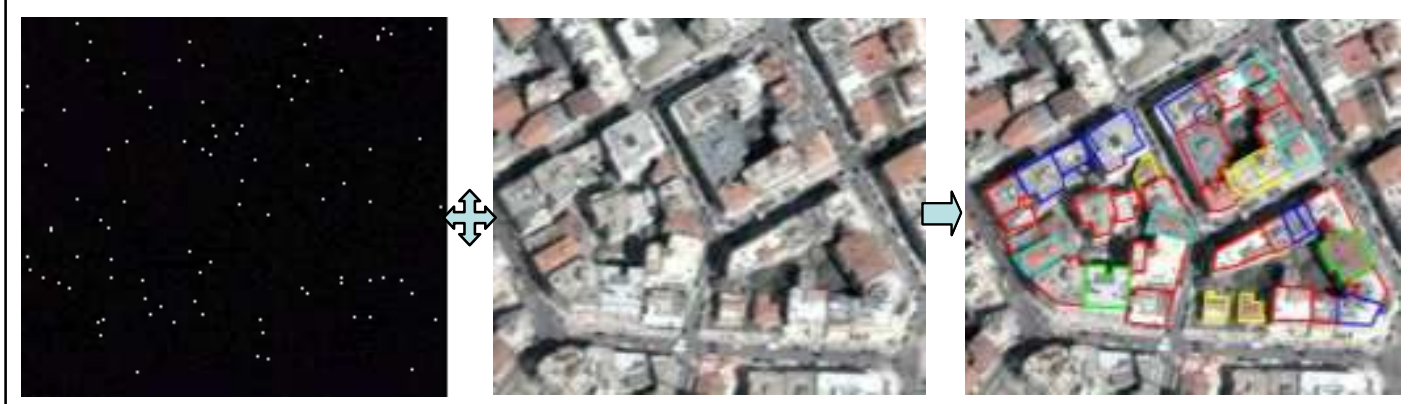

FIGURE 2: Vectorization on satellite image after transformations of translation, scale and rotation.

To do this, the 3D geodetic coordinates $(\varphi, \lambda, h)$ which are given (or can be extracted) from the satellite image of virtual globes such as Google Earth with approximate precision (Nebiker, Christen, Eugster, Flückiger and Stierli, 2007), must be converted previously to adequate coordinates format $(\mathrm{x}, \mathrm{y}, \mathrm{z})$ so that the CAD system can use them. Thus, a project which requires a certain threshold of accuracy and geometric precision must utilize the appropriate photogrammetric methodologies and tools because the coordinates, elevations, distances, and measurements provided by Google Earth are approximations only.

The relationship between the computation of the Cartesian coordinates $x, y, z$ of a given point with its latitude $(\varphi)$, longitude $(\lambda)$ and height $(h)$ are known (Burtch) and they constitute the base on which an algorithm is developed (in Visual Lisp language) in AutoCAD environment in order to convert the geodetic coordinates of the GPS device in Cartesian coordinates (FIG.3).

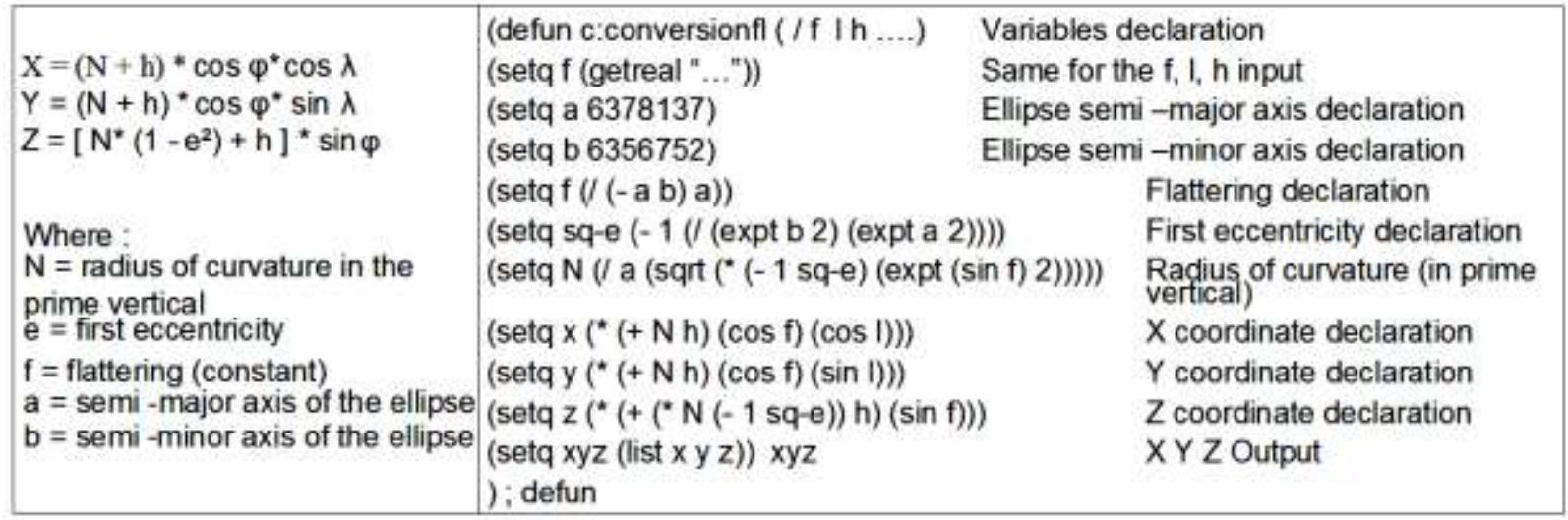

FIGURE 3: Left: Relationship between the computation of Cartesian $(x, y, z)$ and Geodetic coordinates $(\varphi, \lambda, h)$. Right: Algorithm development of the conversion of Geodetic to Cartesian coordinates in Visual Lisp. 


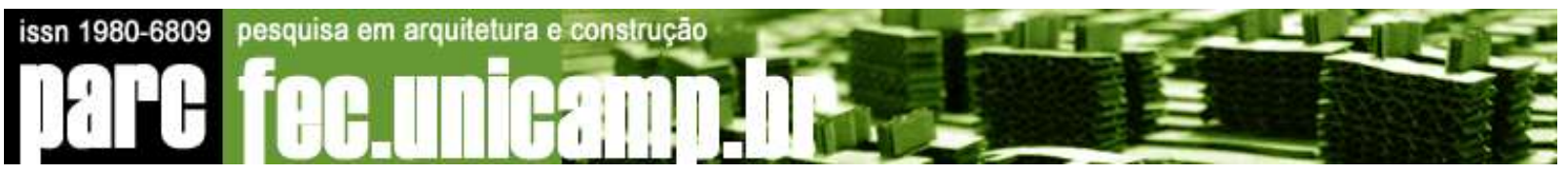

In the fourth step, the orientation of the normals of surfaces must be handled in an appropriate way so that the material assignment could be realized without appearance problems. The operations of orientation and assignment are realized in CAD (AutoCAD) and rendering environment (3D Studio max) respectively, where the "double-faces" surfaces are supported. An interface developed in Visual LISP programming language permits: (a) identification of each surface, (b) visualization of each normal before orientation, (c) surfaces' selection, (d) appropriate reverse of the selected normal and finally (e) visualization of the normals after orientation according to adequate material assignment (FIG.4) (kouzeleas, 2005, 205-210).

\begin{tabular}{|c|c|}
\hline $\begin{array}{l}\text { (defun c:view_normal ( }(. .) \\
\text { (setq ent (entget ...) } \\
\text { (setq normal (...) } \\
\text { (setq p1 p2 p3 p4 (...) } \\
\text { (setq p_normale (list ...) } \\
\text { (if (equal p1 p4) (progn ...) } \\
\text { (setq p_normale1 (...) } \\
\text { (command "_line" ...) } \\
\text { (setq normale_color (entget ...)... } \\
\text { (setq normale_plan (entget ...) } \\
\text { (command "_line" ...)) } \\
\text { (progn ; else } \\
\text { (command "_line" ...)) } \\
\text { (setq normale_color (entget ...)...) } \\
\text { ),if } \\
\text { );defun }\end{array}$ & $\begin{array}{l}\text { Variables declaration } \\
\text { Entities selection } \\
\text { Facette normals calculation } \\
\text { Facettes cordonates } \\
\text { Normal position calculation } \\
\text { Normals detection for } 3 \text { corners faceltes } \\
\text { Normal position calculation for } 3 \text { corners facettes } \\
\text { Normal line draw } \\
\text { Normal color definition } \\
\text { Normal layer definition } \\
\text { Normal extreme orientation line (EOL) definition (draw, color, layer) } \\
\text { Normal position calculation for } 4 \text { corners facettes } \\
\text { Normal line draw } \\
\text { Normal properties definition (color, layer, EOL draw) }\end{array}$ \\
\hline $\begin{array}{l}\text { (defun c:inversion_normal }(/ \ldots \text { ) } \\
\text { (setq ent (entget ...) } \\
\text { (setq couleur (assoc } 62 \text { ent)) } \\
\text { (setq plan (assoc } 8 \text { ent)) } \\
\text { (setq p1 p2 p3 p4 (...) } \\
\text { (command "_erase" ent ".") } \\
\text { (if (equal p1 p4) } \\
\text { (command "_3dface" p1 ...) } \\
\text { (command "-3dface" p1 p4 p3 p2 "'") } \\
\text { (setq ent2 (entget ...) } \\
\text { (entmod (subst ...) } \\
\text { (setq ent3 (entget ...) } \\
\text { (entmod (subst ....)) }\end{array}$ & $\begin{array}{l}\text { Variables declaration } \\
\text { Entities selection } \\
\text { Color filter selection } \\
\text { Layer filter selection } \\
\text { Facette Corners, coordinates definition } \\
\text { Facette erase } \\
\text { If the facette has } 3 \text { comers } \\
\text { Appropriate } 3 \text { corners facette draw } \\
\text { Else appropriate } 4 \text { comers facette draw } \\
\text { Facette assigning properties (color, layer) }\end{array}$ \\
\hline
\end{tabular}

FIGURE 4: Algorithm aspects, representing the surfaces normals selection, visualization and reverse in Visual LISP programming language.

In the fifth step, the Digital Terrain Modeling ("DTM"), representing the vectoring mesh of the relief with approximate accuracy, can be retrieved from special photogrammetric, software in order to adjust it to the satellite imagery (Kraus, Briese, Attwenger, and Pfeifer, 2004, 113118). Special developed algorithms in Visual Lisp interfaces adapted to AutoCAD system can 


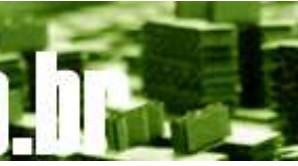

control with accuracy (a) the adjustment of every node of the DTM's vectoring mesh to the corresponding satellite imagery and (b) every bitmap adjustment to the buildings facades (FIG.5); these operations can facilitate the adaptation of the digital architectural constructions to the urban space by corresponding with accuracy coordinates buildings points to vectoring mesh nodes representing the physical urban space.

The DTM is used as the referencing level for the modeling of the buildings in $3 \mathrm{D}$ solid. The simulation of architectural details can be the results of "traditional" vectors modeling with rendering of adequate materials or even the results of assignment of specially edited bitmaps to the buildings facades (kouzeleas, 2008, 205-210).

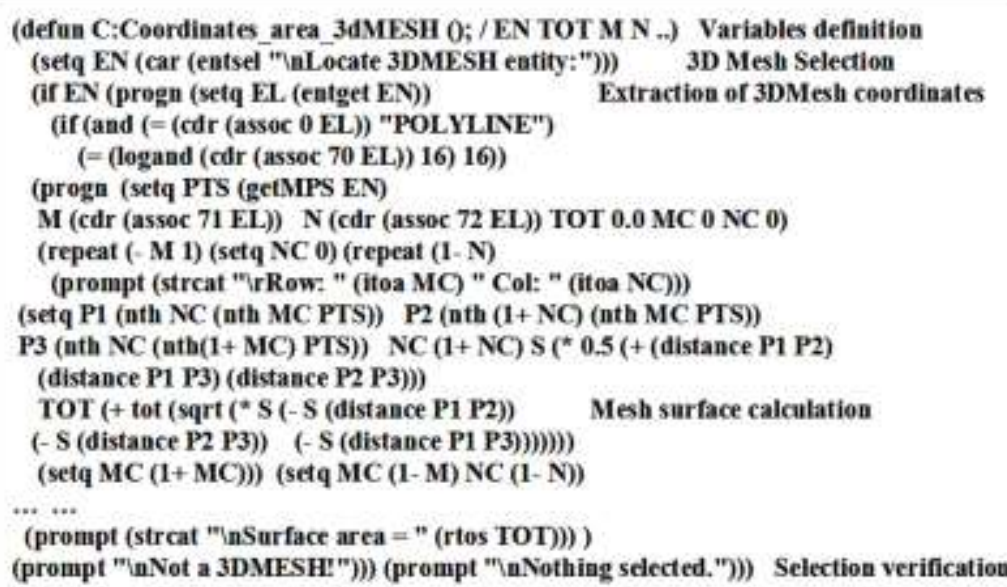

(defua c:DimBITMAP $/$ li wi lm wm rm ri len_adj_perc LengthImagePixel) (setq li (getreal "length of image: $\left.{ }^{\prime \prime} "\right)$ ) (setq wi (getreal "width of image:in")) (setq Im (getdist "leagth of model: $\mathbf{n}^{\text {") }}$ ) (setq wm (getdist "width of model:ln")) (setq $\mathrm{rm}(/ \mathrm{Im} \mathrm{wm}))$; ratio of model's dimension

(setq $\mathrm{ri}(/ \mathrm{ll}$ wi)) ; ratio of image's dimension (setq len_adj perc ( ${ }^{*} 100$; percentage of length image after adjustment

$\left(/\left({ }^{*}\left({ }^{*}\right.\right.\right.$ i wi) $\left.\left.\left.(/ \mathrm{rm} \mathrm{ri})\right)(/(* 1 \mathrm{wi}) \mathrm{D})\right)\right)$ (setq LengthImagePixel ( $/$ li len adj_perc)) ) ; Length of image in Pixel

FIGURE 5: Algorithm view controlling the nodes' coordinates of the DTM vectoring 3Dmesh in Visual Lisp (VL). Right: Algorithm controlling the bitmap dimensions for adjustment to building façade in VL.

In the last step, the simulation and the navigation of the final 3D study area can be realized in AutoCAD, Internet application (“Google Earth") or interactive Virtual Reality ("VR") environment (FIG.6). In AutoCAD, the simulation of satellite imagery, DTM and rendering process creates a slightly similar environment to Google Earth. However, the Google Earth environment permits not only the simple virtual navigation but also the interactive spatial referenced bookmarks of launching multimedia applications (KML, 2011) concerning real images, panorama, sounds or video of the study area. The simulation in VR environment also presents advantages such as the suppleness of navigation and the fullest rendering of the virtual 3D model in parallel with the possibility of interactivity of actions via links through entities ("hyperentities"). The creation of hyperentities is realized via VR authoring and visualization software. 

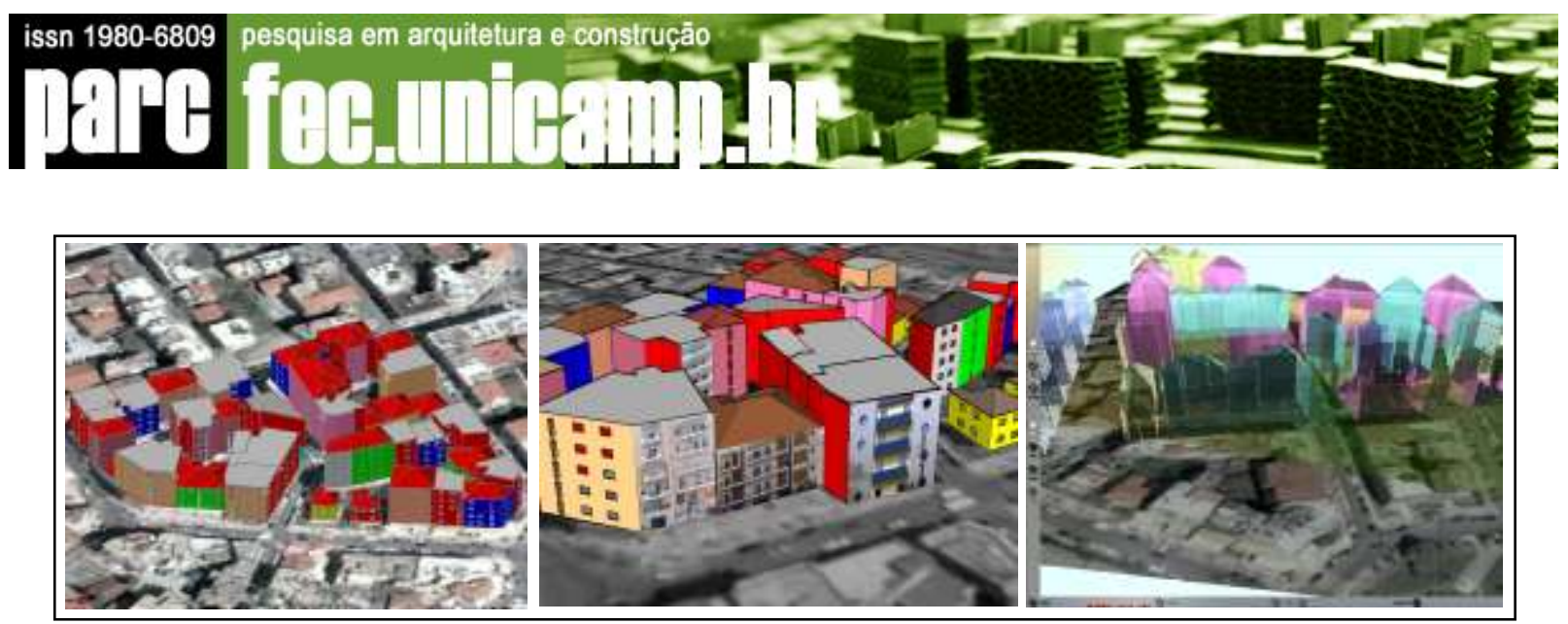

FIGURE 6: Left: 3D solids based on DTM and satellite image in AutoCAD environment. Middle:

Assignment of bitmaps to the buildings facades (Google Earth environment). Right: Virtual Reality environment without rendering (Cortona Vrml Client browser environment) with sound map simulation in superimposed layer to satellite image.

\subsection{Cooperation with automatic modeling process}

This methodology of integrative architectural digital design based on satellite images can cooperate with an automatic modeling process where geographical coordinates and spatial information of selected positions which describe features of the real space are transmitted in real time from the field towards a modeling and simulation system (Papadimitriou, and Kouzeleas, 2005, 98-99).

The function-steps of this system concern the: (a) Positioning, (b) Capturing of descriptive information, (c) Positional and descriptive data transmission, (d) Receiving and registration of spatial data into the system and (e) Simulation in a cartographical environment ("CAD or GIS") (FIG.7); special algorithms and interfaces were developed in order to facilitate the interconnection between composites of two procedures.

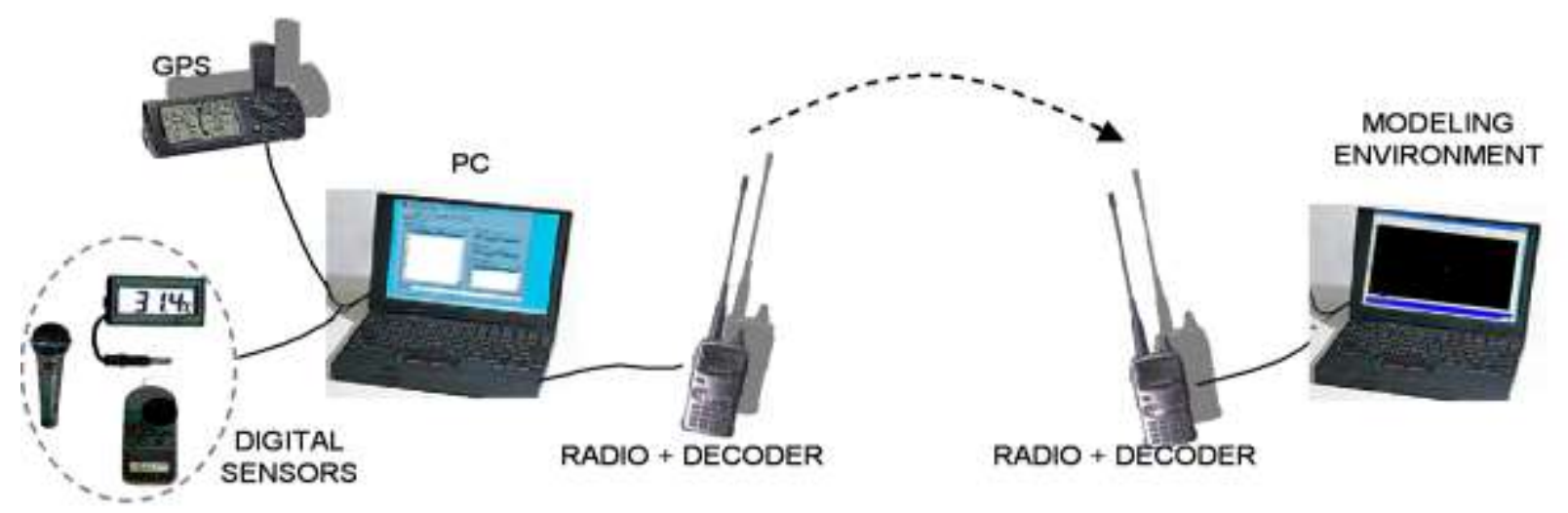

FIGURE 7: Schematic devices connection from the field to the modeling environment. 


\section{Data and technological means}

In the framework of this procedure, computer tools, as they are used in architectural practice today ("professional systems"), or computer tools, which any engineer should be able to use ("social systems"), are used. This "Off-the-shell" software (social systems and professional systems developed by regular software houses) provides basic knowledge of design systems and experience of the use of Computer Aided Architectural Design in practice (Achten, 1996, 7-16).

The available data and technological means for every step of the methodology concern: (a) High resolution imagery and digital terrain model ("DTM") inserted in AutoCAD Architecture environment (a well-known CAD platform which is supported by programming languages), (b) image processing software for the buildings' facades images, (c) Alternative use of Google Earth environment and VR authoring (eg. 3D studio max, Internet Scene Assembler, etc) or visualization software (e.g. Cortona vrml client browser, etc), (d) special algorithms and interfaces development for coordinates conversion, adequate adjustment of bitmaps to the buildings facades and control of the DTM's mesh nodes.

The automatic modeling process which cooperates with the procedure of integrative architectural digital design is based on wide spread technologies such as: (a) handheld GPS device for the position, (b) digital sensors (e.g. microphone, sound pressure meter, thermometer, etc), (c) pair of VHF radio with decoder for the data transmission in real-time, (d) laptop PC in which peripheral devices are connected via serial ports and adequate communication protocols (FIG.7).

\section{Digital design methodologies: related works}

Contemporary techniques of geosciences involving telecommunication means, innovate computational procedures and algorithms, new software and implication of telegeomatic technology are more and more applied in architectural digital design methodologies. The digital simulation of architectural and urban space is supported by dynamic features of Geographical Information System focusing on geomorphologic aspects of space representation (Weis et al, 2005, 311-317). The telegeomatic technology combines spatial decision support systems (“DSS"), GIS and CAD systems with communication processes such as open communication systems ("OCS") integrating new information and 
communication technologies such as Internet, mobile and wireless applications (Boulmakoul, 2006, 514-525). Other digital design methodology use remote monitoring techniques creating real-time applications with networks of sensors in order to capture, and finally simulate coordinates and spatial property measurements (Goni et al, 2009, 141-157). Design processes of a complex building try to use a unique master model as a platform-free software code, in contrast to platform-dependent methods in order to maintain a design logic through implementation of software code from the beginning of schematic design to end of construction document phase (Park, and Holt, 2010, 359-376).

New digital design methodologies use spatial real-time remote monitoring systems (SRTRM) with imagery from satellite and airborne platforms providing digital data in various scales of spatial observation in relation to networks of sensors in order to combine to a unique superimposed multi-layer map geographic coordinates with spatial properties measurements which are hidden from human visual sense, such as sound, degradation intensity light, temperature, humidity, etc. The most common fields of applications of these new digital design methodologies using SRTRM systems concern: (a) Urban land cover, (b) Land change, (c) urban land sprawl, (d) GPS to GIS applications, (e) room environment and (f) spatial growth-analysis. In urban land cover and land use, monitoring of urban settlements via multi-temporal remote sensing imagery has received increasingly greater attention in recent years (Gallego, 2004, 3019-3047) concerning among others changes in land cover, vegetation, spatial texture, classification of data spatial data, etc. Experts systems have been developed where georeferenced information of remotely sensed data such as land use data, spatial texture, and digital elevation models (DEMs) was used to obtain greater classification accuracy using additional spatial datasets such as texture, land use, water rights, city boundaries, and native reservation boundaries (Stefanov et al, 2001, 173-185). In addition, synthetic aperture radar (SAR) and multi-spectral sensors where used for urban area characterization based on multi-spectral and SAR complex images (Gomez-Chova et al, 2006, 234-243). In urban land change, satellite remote sensing and geographic information systems (GIS) have been widely applied in identifying and analyzing land use and land cover change (Ehlers et al, 1990, 175-180). The reference data were collected from field surveys in assisting by Global Positioning Systems (GPS), large-scale aerial photos and satellite images (Wu et al, 2006, 322-333). A variety of techniques used to measure/estimate the area change of the urban form (impervious surfaces), such as digitizing the remote sensing images, remote sensing pattern recognition approaches, such as supervised, unsupervised and knowledge- 
based expert system approaches (Greenberg and Bradley, 1997, 18-22). In architectural and urban land sprawl, the convergence of GIS and database management systems has helped in quantifying, monitoring, modelling, and subsequently predicting the architectural and urban sprawl phenomenon. Statistical techniques, such as multivariate regression, were used to determine the relationship between the percent impervious area and various urban development parameters such as road density, population density, land use type and size of development units (Lo, 2001, 1037-1047). In GPS to GIS application field, the integration of new technologies, such as radio communication permitting wireless interaction between different devices, communication software, GPS devices and GIS applications, helped the development of new systems. These new systems concern, among others, an object-oriented distributed information system for mobile automatic location (OODISMAL) and a Common Object Request Broker Architecture (CORBA) providing the basic components to integrate radio communication and real-time data captured by GPS units with GIS components and the necessary interoperability between devices and software technologies needs (Muro-Medrano et al, 1999, 271-285). In architectural and home environment, monitoring sensors such as humidity sensor, temperature sensor, $\mathrm{CO} 2$ sensor, flying dust sensor, etc., permit to transmit data to PC or PDA devices via specific configuration and software connexion with a web broadcast server (Chung and Oh, 2006, 64-70). All these systems provide supplementary spatial properties information and permit their graphical integration within digital maps of architectural and urban space under development of specific algorithms and integrated digital design methodologies.

Thus, digital design becomes more flexible with the involving of computing media and algorithms search producing new hybrid design workflows in the industry against the industry trend of super-integration software (Derix, 2009, 565-585). However, it is very important to understand the logical relationship of digital design methods and adapt concepts of computable functions to architectural design conception and needs (Kotnik, 2010, 1-16).

\section{Application}

In the context of the 2nd semester course, about fifty engineering students of the Department of urban planning and development engineering of the Aristotle University of Thessaloniki in Greece are introduced to computer aided architectural and urban design; an educational methodology is developed applying to theoretical and practical issues. The educational process is developed multi-dimensionally in three equally important, related 
Pedagogical Thematic Entities concerning: (a) theoretical approach and analysis of a global system of digital, architectural and urban design with related parameters, (b) preparation of the model, presentation and knowledge transfer of the adequate software and (c) final modeling and simulation of the architectural and urban space using innovate techniques and integrating results of computational design researches.

As an experimental field, a central area of an urban space was selected, with a relatively smooth relief next to the everyday living place of the participant students. The urban space provides a geographic background for designing in small (e.g. for urban planning) and great (e.g. for architectural details) engineering scales with rendering techniques of photographic imagery adaptation to buildings facades or rendering texture creation. (FIG. 8).

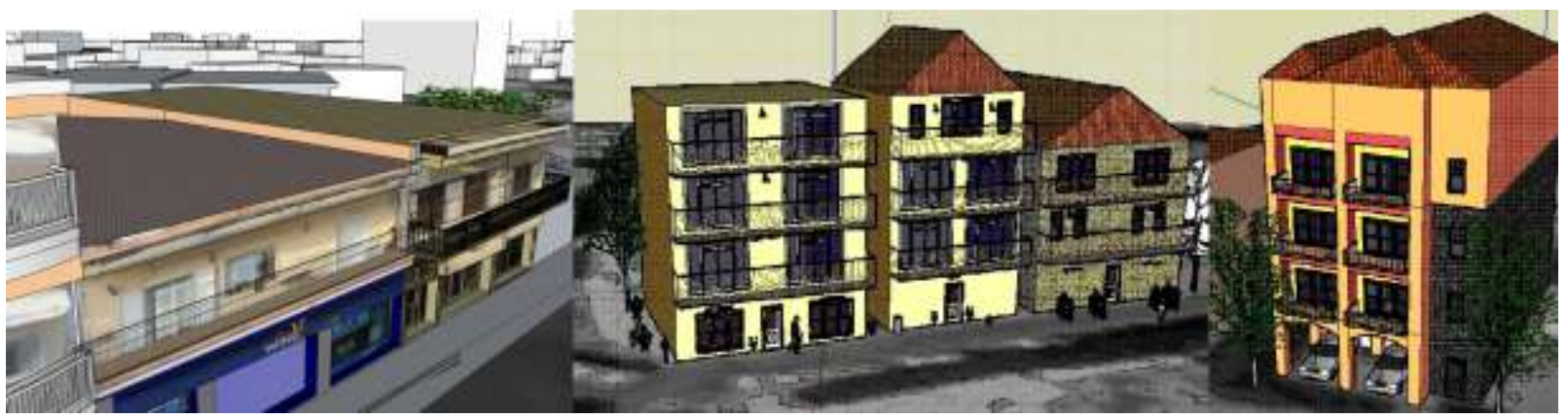

FIGURE 8: Modeling and rendering techniques of bitmaps adaptation to buildings facades or rendering texture use in smooth DTM relief based on satellite image.

The study area was divided to 16 sectors that correspond to 16 work teams. Each work team ( 2 to 4 students) had to carry out four sequential workouts that are combined with specific educational tasks. During the workouts, various pieces of software were used (e.g. CAD platforms supported by programming languages such as AutoCAD Architecture, Google SketchUp; Spatial simulation and navigation platforms such as Google Earth, 3D studio max, and other software concerning image editing and data management operations) providing tools and utilities that are related to the predefined pedagogical thematic entities and their lesson's plans.

The available data for every phase of the methodology included topographic diagrams, satellite images, digital terrain models, high resolution imagery of selected facades and collected additional data of architectural details. The digital built-up area creation followed the above described methodology corresponding: (a) adequate preparation of DTM and satellite images (e.g. coordinates conversion, DTM mesh control, etc), (b) digitalization, (c) 3D 


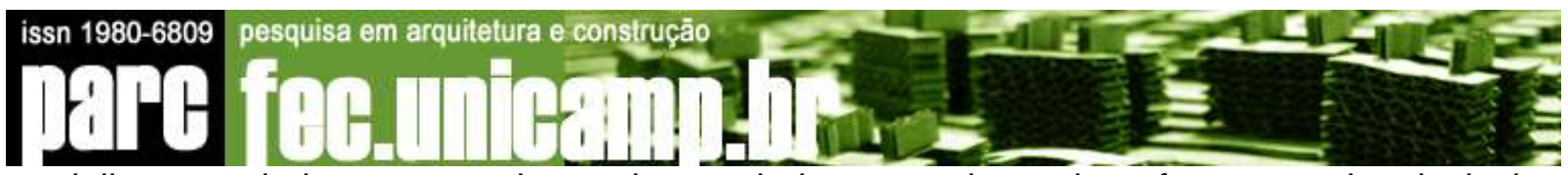
modeling, rendering preparation using techniques and results of computational design procedures (e.g. surfaces normals management, imagery adaptation to buildings facades, etc.), (d) rendering techniques (e.g. photographic imagery use or rendering texture creation) and (e) 3D real-time navigation in interactive environment (e.g. Google Earth, 3D studio max animation, Virtual Reality and multimedia platforms) with spatial properties simulation (e.g. sound map) in superimposed layer to satellite image (FIG. 9)
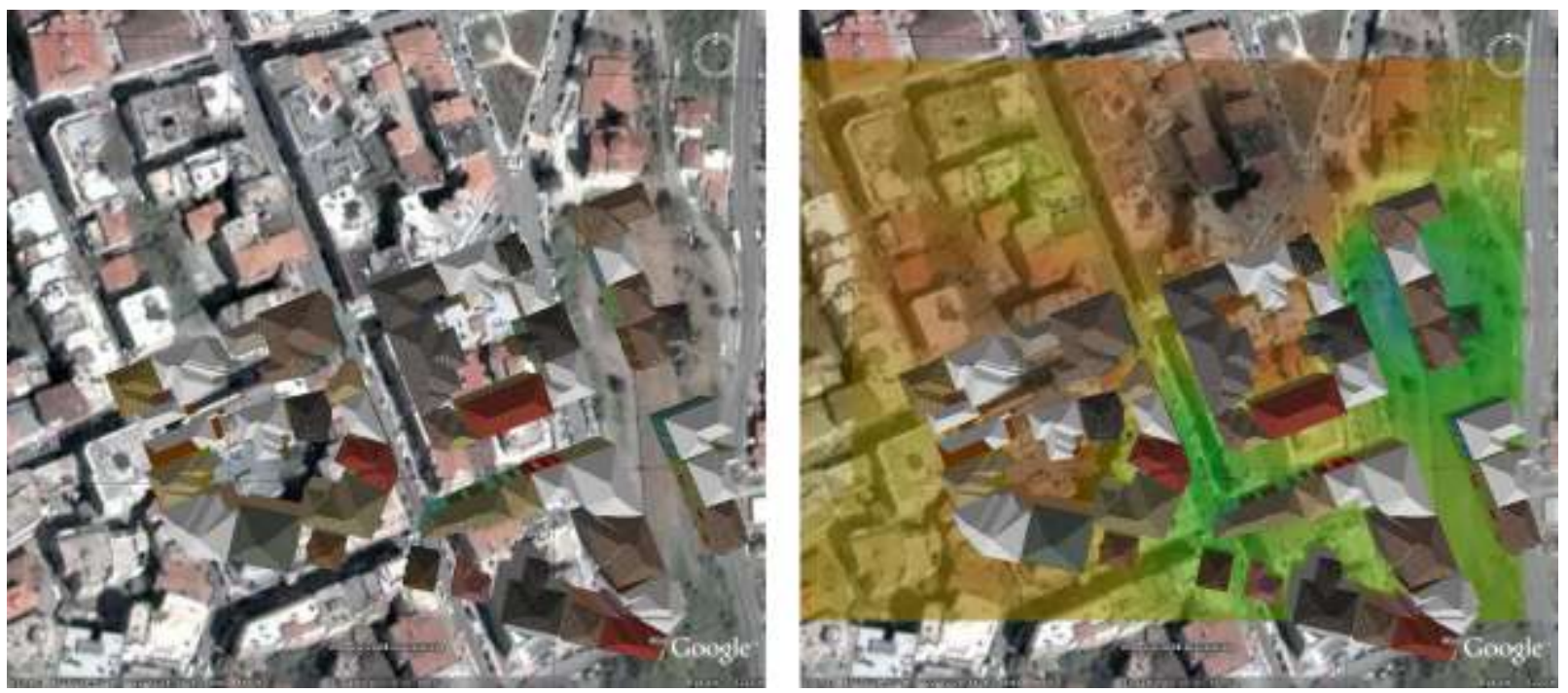

FIGURE 9: Digital architectural simulation with superimposed sound map layer (using XML programming techniques) based on satellite image in interactive navigation environment (GoogleEarth or Virtual Reality)

The participants-students to this study had no previous experience of digital design and design methodology. Their introduction to theoretical and practical digital design issues was for many of them a difficult process. In the begging of the courses, the students were not able to understand the relation between theory and practice and how they could arrive at this level of results without previous experience and knowledge. The fact that they were in groups helped them significantly to be adapted, to understand better the methodology and share a common experience. From the midway of the courses and after, the majority of the students started to express their positive impressions because of the more practical nature of the process. In the end of the courses the results started to become more and more visible (e.g. 3D modeling, imagery integration in buildings facades, digital city's sectors consolidation, etc) and the students started to be very enthusiastic and available. The grade results encourage the goals of this educational digital design process and show that $76 \%$ of students in 
theoritical exams and $67 \%$ of them in practical exams succeded (FIG. 10). Precisely, in theorital exams, $24 \%$ of the students had a bad mention ( 0 to 5 grade in the scale of 10 ), $55 \%$ of them a good to very good mention ( 5 to 8 grade) and finally $21 \%$ of them an excellent performance (8 to 10 grade). In practical exams, 33\% of the students had a bad mention (0 to 5 grade in the scale of 10 ), $43 \%$ of them a good to very good mention (5 to 8 grade) and finally $24 \%$ of them an excellent performance ( 8 to 10 grade). Obviously, for the students the practical issues were more difficult than the theorical. The performance of the students could be more significant if they attended higher academic level where other courses of urban design, geoinformatics and design analysis help them to improve their spatial perception and analysis.

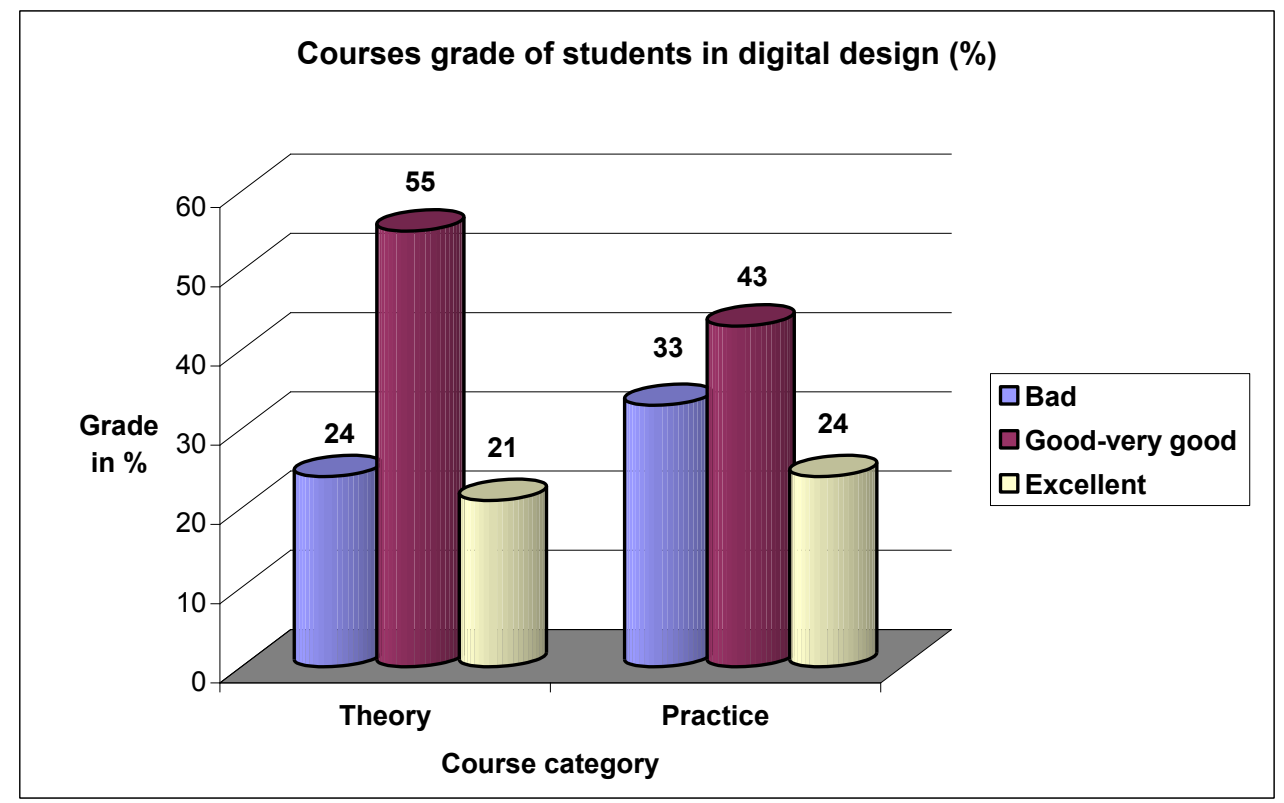

FIGURE 10: Grade in theoretical and practical issues (in \%) of students courses in digital design

\section{Conclusions}

The procedure of architectural digital design is based on satellite images as geographical background reference in order to position the vectoring models. The application of transformations is necessary so that every coordinate, dimension and distance correspond to the real one. The automation of the conversion from geodetic to cartesian coordinates, the adjustment of the bitmaps dimensions in order to adjust them adequately to buildings facades, the control of the surfaces normal in order to facilitate the rendering and finally the possibility of controlling every node of the DTM's 3D mesh add more accuracy, velocity to the methodology. Additionally, these specific interfaces are developed in a CAD system 
("AutoCAD") which is supported by a powerful tool of programming. Thus, a Computer Aided

Architectural Design tool turns one's time to account innovate techniques and technological means which are used in other photogrammetric applications and disciplines in order to give to the architectural digital design perspectives of contemporary multidisciplinary adaptation.

The limitations of the abovementioned integrative architectural and urban digital design methodology are restricted to the :

(a) complex synergy of different tools

(b) specific competence in knowledge in different disciplines (digital architectural and urban design, topography, computers, etc)

(c) interfaces development for function and environment connection and tool adaptation in special needs.

The combination of (a) new widespread developing technologies, (b) existent digital design and interactive spatial simulation and navigation systems in real-time with capability of internet links with multimedia applications (e.g. GoogleEarth, Virtual Reality environment), (c) complementary spatial properties simulation of superimposed digital cartography with geographic reference (e.g. sound, temperature, humidity, etc), and finally (d) the possibility of programming language development for tools adaptation in user's needs and environments, with the procedure of architectural and urban digital design gives opportunity to future developments and easier adaptation in evolution perspectives. Future developments of the proposed methodology concern, among other:

(a) digital cities creation in architectural or urban scale,

(b) study of existent or previsional urban situation with spatial statistic or qualitative indicators creation (covering construction area, free public area, green area, road network, etc),

(c) study of digital simulation of urban sprawl,

(d) creation of holistic environment simulation (geographic space and spatial properties) with alternative activities propositions, special conditions zones definition, redefinition of "land use" zones related to superimposed digital cartography of spatial properties with geographic reference,

(e) identification, comparison, thematic classification and correlation of spaces in relation to their spatial properties,

(f) eventual objective criteria introduction of space's thematic classification based on geographic and descriptive property of every taken spatial property (e.g. quality indicators specification, production methods evolution, spatial impact indicators, etc). 


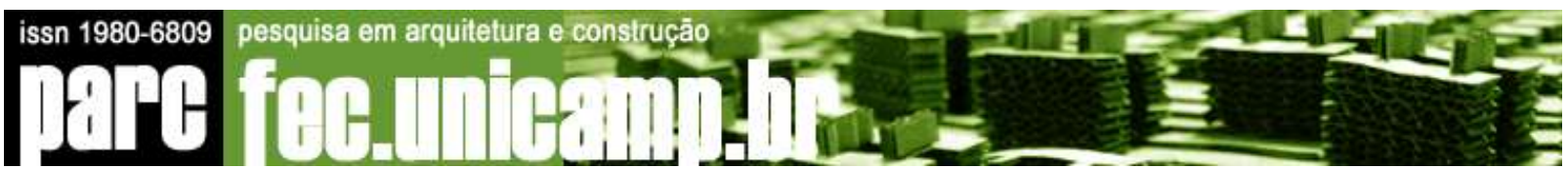

The proposed integrative architectural and urban digital design methodology with all its computational design contributions and perspectives, gives the opportunity of an holistic approach, description and undestanding of space architecture where the access in information and in spatial perception can be "more democratic" and participative with a view a personnal interpretation and comprehension of the space from each user.

\section{References}

Achten, Henri. "Teaching advanced Architectural issues through principles of CAAD. "Proceedings of 14th eCAADe Conference, Lund Sweden, 1996. 7-16. Print.

Boulmakoul Abdel. "Fuzzy graphs modeling for HazMat telegeomonitoring." European Journal of Operational Research 175.3 (2006) : 514-525. Print

Burtch, Robert. "A comparison of methods used in rectangular to geodetic coordinate transformations." Proceeding of American Congress on Surveying and Mapping (ACSM'06). Orlando Florida USA, 2006.

Chung, Wan-Young and Sung-Ju Oh. "Remote monitoring system with wireless sensors module for room environment." Sensors and Actuators B: Chemical 113.1 (2006) : 64-70. Print.

Derix, Christian. "In-Between Architecture Computation." International journal of architectural computing 7.4 (2009) : 565-585. Print

Ehlers, Jadkowski, et al. "Application of SPOT data for regional growth analysis and local planning." Photogrammetric Engineering \& Remote Sensing 56 (1990) : 175-180. Print.

Gallego, F. Javier. "Remote sensing and land cover area estimation." International Journal of Remote Sensing 25 (2004) : 3019-3047. Print.

Gomez-Chova, Fernandez-Prieto, et al. "Urban monitoring using multi-temporal SAR and multi-spectral data." Pattern Recognition Letters 27.4 (2006) : 234-243. Print.

Goni, Burgos, et al. "Architecture, cost-model and customization of real-time monitoring systems based on mobile biological sensor data-streams" Computer Methods and Programs in Biomedicine 96.2 (2009) : 141-157. Print

Greenberg, J.D. and G.A. Bradley. "Analyzing the urban-wildland interface with GIS." Journal of Forestry 95 (1997) : 18-22. Print

Kouzeleas, Stelios. "Processes aspects of modeling, interactive digital visualization and multimedia representation of architectural and urban area." Proceedings of 11th International Conference on Computer Graphics and Artificial Intelligence (3IA'2008), Athens Greece, 2008. 205-210. Print.

Laurini, Robert. "A Short Introduction to TeleGeoProcessing and TeleGeoMonitoring." Proceedings of 2nd International Symposium On Telegeoprocessing (TeleGeo 2000). Nice-Sophia-Antipolis France, 2000. 1-12. Print. 
Lo, C.P. "Modeling the population of China using DMSP operational Linescan system nighttime data."

Photogrammetric Engineering \& Remote Sensing 67 (2001) : 1037-1047. Print.

KML. "KML Reference." Google code. 2011. Web. 18 July 2011

<http://code.google.com/intl/el/apis/kml/documentation/kmlreference.html>.

Kotnik, Toni. "Digital Architectural Design as Exploration of Computable Functions." International journal of architectural computing 8.1 (2010) : 1-16. Print

Kraus, Briese, et al. "Quality measures for digital terrain models." Proceedings of XXth ISPRS Congress, Istanbul Turkey, 2004. 113-118. Print.

Muro-Medrano, Infante, et al. "A CORBA infrastructure to provide distributed GPS data in real time to GIS applications." Computers Environment and Urban Systems 23.4 (1999) : 271-285. Print.

Nebiker, Christen, et al. "Integrating mobile geo sensors into collaborative virtual globes-design and implementation issues." Proceedings of 5th International Symposium on Mobile Mapping Technology (MMT'07). Padua Italy, 2007.

Papadimitriou, Kimon and Stelios Kouzeleas. "A method for real time spatial analysis of sound via modeling in a CAD environment based on acoustical measurements." Proceedings of 14th European Colloquium on Theoretical and Quantitative Geography, Tomar Portugal, 2005. 98-99. Print.

Park, Kat and Nicholas Holt. "Parametric Design Process of a Complex Building In Practice Using Programmed Code As Master Model." International Journal of Architectural Computing 8.3 (2010) : 359-376. Print.

Stefanov, Ramsey, et al. "Monitoring urban land cover change: An expert system approach to land cover classification of semiarid to arid urban centers." Remote Sensing of Environment 77.2 (2001) : 173-185. Print.

Tanzi, Tullio Joseph. "Principles and Practices in TeleGeomatics." Proceedings of 2nd International Symposium On Telegeoprocessing (TeleGeo 2000). Nice-Sophia-Antipolis France, 2000. 13-24. Print.

Weis, Müller, et al. "A framework for GIS and imagery data fusion in support of cartographic updating" Information Fusion, 6.4 (2005) : 311-317. Print.

$\mathrm{Wu}$, Li, et al. "Monitoring and predicting land use change in Beijing using remote sensing and GIS." Landscape and Urban Planning 78.4 (2006) : 322-333. Print. 